\section{P078 VIRAL LOAD MONITORING FOR PEOPLE LIVING WITH HIV IN THE ERA OF TEST AND TREAT - AN UPDATED SYSTEMATIC REVIEW}

${ }^{1,2} \mathrm{M}$ Pham*. ${ }^{1}$ Burnet Institute, Melbourne, Australia; ${ }^{2}$ Monash University, Melbourne, Australia

10.1136/sextrans-2021-sti.210

Background Treatment of HIV with antiretroviral therapy (ART) can save lives and stop the spread of the virus. In 2014, UNAIDS launched the 90-90-90 treatment target. By 2020, it remains unattainable in many low and middle-income countries (LMICs). This review aimed to identify research gaps and needs for interventions to improve viral load monitoring and viral suppression for people living with HIV (PLHIV) in LMICs.

Methods Medline and PubMed were searched to identify relevant literature, published in English between Dec 2015 and May 2020, using key search terms of a review published in 2016. The primary outcome was initial viral load (VL) monitoring (the proportion of PLHIV on ART and eligible for VL monitoring who receive a VL test). Secondary outcomes include follow-up VL monitoring (the proportion of PLHIV who receive a follow-up VL after an initial elevated VL test), confirmation of treatment failure (the proportion of PLHIV who had two consecutive elevated VL test results) and switching treatment regimen rates (the proportion of PLHIV switching treatment regimen after confirmation of treatment failure). Results The search identified 1829 non-duplicate records, of which 23 were included in the review. More than 80\% (19/ 23) of included studies were conducted in 11 sub-Saharan African countries (SSA) and most were published in 20192020. Marked variations in initial VL monitoring coverage were reported across study settings (11-93\%) and study populations (adults (25-93\%), children and adolescents (2-94\%), and pregnant women (32-67\%)). Suboptimal uptake of follow-up VL monitoring and low regimen switching rates after confirmed treatment failure were observed.

Conclusions Substantial gaps in VL coverage across study settings and study populations remained evident with limited data availability outside of SSA. Further research is needed to fill the data gaps. Interventions to address the 'failure cascade' in PLHIV on ART who fail to achieve viral suppression are required.

\section{P079 SYPHILIS REINFECTION AMONG WOMEN: FLORIDA AND LOUISIANA, USA 2000-2018}

'D Newman* ${ }^{*}$, $2 \mathrm{~J}$ Matthais, ${ }^{1} \mathrm{M}$ Rahman, ${ }^{3,4} \mathrm{~A}$ Brantley, ${ }^{1} \mathrm{~T}$ Peterman. ${ }^{1} \mathrm{CDC}$, Atlanta, USA; ${ }^{2}$ Florida Department of Health, Tallahassee, USA; ${ }^{3}$ Louisiana Department of Health, New Orleans, USA; ${ }^{4}$ Michigan Department of Health and Human Services, Lansing, USA

\subsection{6/sextrans-2021-sti.211}

Background Syphilis rates have continued to rise in the United States. Florida and Louisiana consistently report high numbers of cases. In a previous study, reinfections accounted for $30.4 \%$ of new infections among men in 2018. In this analysis, we evaluated rates of reported reinfections in women.

Methods We evaluated all syphilis records, all stages (primary, secondary, early latent, and late latent) in females aged 15-70 years from the Florida and Louisiana Departments of Health surveillance databases during 2000-2018. Demographics of cases and repeaters (individuals reported with 2 or more cases of syphilis) were examined. Percentages of syphilis cases from repeaters by year were calculated as were percentages from HIV + females.

Results From 2000-2018, 124,827 syphilis cases were reported from 107,405 individuals: 33,594(31\%) were female accounting for $35,279(28 \%)$ of all cases. Of 33,594 females, 32,050 (95\%) reported a single episode of syphilis and 1,544(5\%) were repeaters. Most women with a single case report were African American 18,429(57.5\%) and 1,510(4.7\%) of single cases were HIV+. Women with a single episode of syphilis were likely in the age range of 20-39 (primary child-bearing years) $20,037(63 \%)$. Repeaters were primarily African American 914(59.2\%) and 218(14.1\%) were HIV+. Women reported with two or more episodes of syphilis also were likely to be of child-bearing age 20-39: 1,065(69\%). Repeaters had 1,685 cases reported; range 2 to 5 . Mean time to second infection was 3.5 years (median $=2.3$ years). From 2010 2018, repeaters accounted for a slowly increasing percentage of all syphilis reported: 2010 (5.1\%), 2013 (5.9\%), 2016 $(6.8 \%)$, and reached $(10.3 \%)$ in 2018. Within females living with HIV, cases from repeaters also increased: 2010 (13\%), 2014 (16\%), 2016 (21\%), and 2018 (25\%).

Conclusion Most syphilis diagnosed in Florida and Louisiana was among persons infected for the first time and among men. However, syphilis infection and reinfections are increasing in women.

\section{P080 THE ROLE OF EXOGENOUS SEX STEROIDS ON THE VAGINAL MICROBIOTA: A SYSTEMATIC REVIEW}

${ }^{1,2} \mathrm{~L}$ Ratten, ${ }^{1,2} \mathrm{E}$ Plummer, ${ }^{1,2,3} \mathrm{C}$ Bradshaw, ${ }^{1,2} \mathrm{C}$ Fairley, ${ }^{4,5,6} \mathrm{~S}$ Garland, ${ }^{4,5,6} \mathrm{G}$ Murray, ${ }^{7,8,9} \mathrm{G}$ Tachedjian, ${ }^{1,2,3} \mathrm{~L}$ Vodstrii ${ }^{*} .{ }^{1}$ Central Clinical School, Monash University, Melbourne, Australia; ${ }^{2}$ Melbourne Sexual Health Centre, Alfred Health, Carlton, Australia; ${ }^{3}$ Melbourne School of Population and Global Health, The University of Melbourne, Parkville, Australia: ${ }^{4}$ Women's Centre for Infectious Diseases, The Royal Women's Hospital, Parkville, Australia; ${ }^{5}$ Murdoch Children's Research Institute, Parkville, Australia; ${ }^{6}$ Department of Obstetrics and Gynaecology, The University of Melbourne, Parkville, Australia; 'Burnet Institute, Melbourne, Australia; ${ }^{8}$ Department of Microbiology, Monash University, Clayton, Australia; ${ }^{9}$ Department of Microbiology and Immunology, University of Melbourne, Melbourne, Australia

\subsection{6/sextrans-2021-sti.212}

Background There is considerable interest globally in understanding the influence of exogenous sex steroids/hormones on the vaginal microbiota (VM). We conducted a systematic review summarising the influence of hormonal contraception (HC) and hormone replacement therapy (HRT) on the VM among reproductive-aged women and peri/post-menopausal women, respectively. PROSPERO registration: CRD42018107730.

Methods To be eligible for inclusion, studies had to report on sex steroid use (HC or HRT) and provide a measurement of the VM by molecular methods. Two authors systematically identified and extracted data from eligible studies. Data regarding the 'positive', 'negative' or 'neutral' effect of exogenous sex steroid use on the VM was summarised. A positive effect was defined as an increased abundance of lactobacilli, decreased abundance of bacteria associated with a non-optimal VM, decreased bacterial diversity and/or increased bacterial stability, relative to partcipants' pre-treatment specimen or compared to a non-exposed control population. A negative effect was defined as parameters in the opposite direction, and a neutral effect was defined as no specific effect identified. 